\title{
History of acoustic neutrino detection
}

\author{
Robert Lahmann ${ }^{1, \star}$ \\ ${ }^{1}$ Friedrich-Alexander-Universität Erlangen-Nürnberg, Erlangen Centre for Astroparticle Physics, Erwin- \\ Rommel-Str. 1, 91058 Erlangen, Germany
}

\begin{abstract}
The historic development of acoustic neutrino detection is shortly reviewed from early ideas starting in the 1950s to current experiments. The article concludes with an assessment of the current status of the field and outlook to the future, as perceived by the author, and with an emphasis on activities in the Mediterranean Sea.
\end{abstract}

\section{A historic introduction to acoustic neutrino detection}

In 1957, G.A. Askariyan pointed out that ionisation and cavitation along the track of an ionising particle in a liquid leads to hydrodynamic radiation [1]. At that time, the detection of the neutrinopublished in July 1956 [2] — had been recent news and the acoustic detection of these new particles was not yet a subject. State of the art for particle detection was the bubble chamber. In search for novel detection methods, piezo sensors irradiated with high energy electron beams were shown to emit sound waves and the potential for detecting individual particle tracks with this method was discussed [3].

Starting in the 70's, theoretical and experimental studies on the hydrodynamic radiation of beams and particles traversing liquids [4-10] were conducted. These efforts lead to the formulation of the so-called thermo-acoustic model [4], according to which the energy deposition of particles traversing liquids (or other suitable dense media) leads to a local heating of the medium which can be regarded as instantaneous with respect to hydrodynamic time scales. Due to the temperature change, the medium expands or contracts according to its bulk volume expansion coefficient $\alpha$. The accelerated motion of the heated medium generates an ultrasonic pulse whose temporal signature is bipolar and which propagates in the volume. The effect has been confirmed in the meantime by a number of laboratory experiments (see e.g. [11] and references therein) with particle beams and lasers.

The acoustic detection of cosmic, i.e. astrophysical neutrinos was discussed within the DUMAND neutrino detector project [12] and first ideas were presented at the 1976 DUMAND workshop independently by T. Bowen and B.A. Dolgoshein [13] and subsequently published [14, 15]. For DUMAND, the energy threshold for acoustic detection, thought to be in the region of $10^{16} \mathrm{eV}$, was eventually considered too high ${ }^{1}[12]$ and the emphasis was put on the detection of Cherenkov light from charged particles emerging from neutrino interactions. It is noteworthy that DUMAND, just like current-day underwater Cherenkov neutrino telescopes, was planned with an acoustic position calibration system and a dual use of this system for positioning and neutrino detection was proposed [17].

\footnotetext{
^e-mail: robert.lahmann@fau.de
}

${ }^{1}$ In two earlier publications from 1977 , a threshold of $10^{13} \mathrm{eV}$ for large scale acoustic water detectors is mentioned [14, 16]. 
With acoustics being sidelined in DUMAND, the $\mathrm{SADCO}^{2}$ collaboration formed, which looked for alternative sites for acoustic neutrino detection. In this context, the use of existing arrays, in particular military arrays, was discussed [18]. A sonar facility with an array of 2400 hydrophones in the Pacific Ocean near the Kamchatka Peninsula was considered as an option. Another array, the MG$10 \mathrm{M}$ system, with 132 hydroacoustic sensors arranged as a cylinder of $1 \mathrm{~m}$ height and $1.6 \mathrm{~m}$ diameter, was taken out of service by the USSR Navy and was sensitive in a frequency range better suited for acoustic neutrino detection. Projects involving these arrays were however not realised and during the final phase of the DUMAND project, the NESTOR project in the Mediterranean Sea emerged. In the context of SADCO, the deployment of an acoustic array at the NESTOR site was discussed [19].

In 1995, further efforts on DUMAND were cancelled by the US DOE [20]. With ANTARES in the Mediterranean Sea, AMANDA in Antarctica and the Lake Baikal experiment in Siberia, Cherenkov neutrino telescopes eventually became a reality. Further activities in acoustic neutrino detection, pursued as an option to extend neutrino telescopes to larger volumes, will be discussed in Section 3 .

\section{Physics aspects of acoustic detection}

A thermo-acoustic wave, generated by heating a suitable medium, can be described by the pressure difference $p^{\prime}=p-p_{0}$ between the ambient pressure $p$ and the equilibrium pressure $p_{0}$, yielding the relation $[4,5]$

$$
\nabla^{2} p^{\prime}-\frac{1}{c_{s}^{2}} \frac{\partial^{2} p^{\prime}}{\partial t^{2}}=\frac{\partial^{2} \epsilon}{\partial t^{2}}
$$

with the adiabatic sound velocity $c_{s}$ and the energy deposition density $\epsilon$. This formulation assumes an isotropic energy deposition without momentum transfer to the medium [11]. Inhomogeneous wave equations equivalent to Eq. (1) have also been derived using the velocity potential [21] and the acoustic displacement potential [15], respectively, instead of the pressure $p^{\prime}$ as function in the differential equation. The general solution to Eq. (1) for the pressure difference measured at time $t$ and position $\mathbf{r}$ is the Kirchhoff integral [22]

$$
p^{\prime}(\mathbf{r}, t)=\frac{1}{4 \pi} \frac{\alpha}{c_{p}} \int_{V} \frac{\mathrm{d} V^{\prime}}{\left|\mathbf{r}-\mathbf{r}^{\prime}\right|} \frac{\partial^{2}}{\partial t^{2}} \epsilon\left(\mathbf{r}^{\prime}, t-\frac{\left|\mathbf{r}-\mathbf{r}^{\prime}\right|}{c_{s}}\right) .
$$

with the aforementioned bulk volume expansion coefficient $\alpha$ and the specific heat capacity at constant pressure $c_{p}$. The position vectors $\mathbf{r}^{\prime}$ expand over the heated volume.

The local heating due to the energy deposition by a hadronic cascade can be regarded as instantaneous with respect to the hydrodynamic time scales. Hence the temporal dependence of $\epsilon$ can be approximated by a step function, whose time derivative yields a delta function. Substantial differences between the peak amplitudes of the acoustic signals for different calculations, as e.g. reported in [23], stem mostly from the assumptions about the spatial energy distribution $\epsilon\left(\mathbf{r}^{\prime}\right)$ in the shower. More recently, the CORSIKA program, designed to simulate extensive cosmic ray air showers, has been adapted to simulate energy depositions in water or ice, then using a numerical calculation based on a formula equivalent to Eq. (2) to simulate the thermo-acoustic pressure signal at a given position [24].

Coherent superposition of the elementary sound waves, produced over the cylindrical volume of the energy deposition, leads to a propagation within a flat disk-like volume in the direction perpendicular to the axis of the particle shower. The peak frequency of the sound signal is governed by the lateral extension of the shower and is expected to be around $f_{\text {peak }} \approx c_{s} / d_{\text {shower }} \approx 15 \mathrm{kHz}$ with $c_{s} \approx 1.5 \mathrm{~m} / \mu$ s for a shower diameter of $d_{\text {shower }} \approx 10 \mathrm{~cm}$.

\footnotetext{
${ }^{2}$ Sea-based Acoustic Detector of Cosmic Objects
} 
Table 1. Conferences and workshops on detection of neutrinos using acoustic and radio techniques since the beginning of the century.

\begin{tabular}{lllcr}
\hline \hline year & name & location & participants & $\begin{array}{r}\text { acoustic } \\
\text { talks }\end{array}$ \\
\hline \hline 2000 & RADHEP & Los Angeles & 50 & 1 \\
2003 & Acoustic mini workshop & Stanford & 20 & 16 \\
2005 & ARENA2005 & Zeuthen & 90 & 26 \\
2006 & ARENA2006 & Newcastle & 50 & 13 \\
2008 & ARENA2008 & Roma & 80 & 22 \\
2010 & ARENA2010 & Nantes & 80 & 12 \\
2012 & ARENA2012 & Erlangen & 70 & 10 \\
2014 & ARENA2014 & Annapolis & 60 & 5 \\
2016 & ARENA2016 & Groningen & 55 & 10 \\
2018 & ARENA2018 & Catania & 60 & 10 \\
\hline \hline
\end{tabular}

Acoustic detection has also been discussed in other dense media, in particular in ice, salt $[25,26]$ and permafrost [27]. While acoustic test setups in ice are part of the discussion in the next section, acoustic activities in salt and permafrost have not been reported since the aforementioned publications.

\section{Acoustic particle detection activities in the new century}

A new push of acoustic particle detection activities following those described in Section 1 roughly coincides with the beginning of the new century. Acoustic detection-together with the detection of radio signals [28]—of ultra-high energy $\left(E \gtrsim 10^{18} \mathrm{eV}\right)$ neutrinos was rediscovered as a promising method to extend the energy scale beyond that reachable with Cherenkov neutrino telescopes. Motivation has been the expected flux of ultra-high energy "cosmogenenic" neutrinos, sometimes called a "guaranteed flux", produced in the reaction of ultra-high energy cosmic rays with the cosmic microwave background [29-31]. The detection of such neutrinos is considerably more challenging than the search for high-energy neutrinos $\left(E \gtrsim 10^{10} \mathrm{eV}\right)$ as currently pursued by under-ice and underwater Cherenkov neutrino telescopes [32-34]. Due to the low expected fluxes, detectors with effective volumes exceeding $100 \mathrm{~km}^{3}$ are needed [35]. However, the properties of the acoustic method allow for sparsely instrumented arrays with $\sim 100$ sensors $/ \mathrm{km}^{3}$. In table Table 1, a list of conferences and workshops since the beginning of the century is given, in which radio and acoustic particle detection techniques-both targeting ultra-high energy neutrinos—-have been the principal subjects.

Most current or recent test setups for acoustic neutrino detection have either been add-ons to optical neutrino telescopes in water or ice, or have been using existing acoustic submarine arrays built for other purposes, typically for military use. Their acoustic sensors are based on the piezoelectric effect [36]. An overview of these experiments in sea water, fresh water and ice, currently taking data or having done so until recently, is given in Table 2. Details on the experiments can be found in the references given in the table.

At the time of writing of this article, SPATS, SMO and the Lake Baikal experiment were still taking data ${ }^{3}$, while the KM3NeT infrastructure [46] is under construction in the Mediterranean Sea. It will comprise a huge array of acoustic sensors for position calibration that can be used for the next step of studies of acoustic neutrino detection [45]. Concepts employing fibre-based hydrophones

\footnotetext{
${ }^{3}$ For the military arrays, this means that data are not used for investigations of neutrino detection methods any more
} 
Table 2. Overview of existing and recent acoustic detection test sites.

\begin{tabular}{lllll}
\hline \hline Experiment & Location & Medium & $\begin{array}{l}\text { Sensor } \\
\text { Channels }\end{array}$ & $\begin{array}{l}\text { Host } \\
\text { Experiment }\end{array}$ \\
\hline \hline SPATS [37, 38] & South Pole & Ice & 80 & IceCube \\
\hline Lake Baikal [39] & Lake Baikal & Fresh Water & 4 & $\begin{array}{l}\text { Baikal Neutrino } \\
\text { Telescope }\end{array}$ \\
\hline OvDE [40] & Mediterranean Sea (Sicily) & Sea Water & 4 & NEMO \\
\hline AMADEUS [41] & Mediterranean Sea (Toulon) & Sea Water & 36 & ANTARES \\
\hline ACoRNE [42] & North Sea (Scotland) & Sea Water & 8 & Rona military array \\
\hline SAUND [43] & $\begin{array}{l}\text { Tongue of the Ocean } \\
\text { (Bahamas) }\end{array}$ & Sea Water & $7 / 49^{(\star)}$ & $\begin{array}{l}\text { AUTEC military } \\
\text { array }\end{array}$ \\
\hline $\begin{array}{l}\text { SMO } \\
\text { sensor array [44] }\end{array}$ & $\begin{array}{l}\text { Mediterranean Sea } \\
\text { (Sicily) }\end{array}$ & Sea Water & 10 & $\begin{array}{l}\text { NEMO Phase-II } \\
\text { prototype }\end{array}$ \\
\hline $\begin{array}{l}\text { KM3NeT } \\
\text { sensor array [45] }\end{array}$ & $\begin{array}{l}\text { Mediterranean Sea } \\
\text { (Sicily, Toulon) }\end{array}$ & Sea Water & $\begin{array}{l}\text { under } \\
\text { constr. }\end{array}$ & KM3NeT \\
\hline \hline \multirow{2}{*}{ The number of hydrophones was increased from 7 in SAUND-I to 49 in SAUND-II } & \\
\hline
\end{tabular}

${ }^{(\star)}$ The number of hydrophones was increased from 7 in SAUND-I to 49 in SAUND-II

as an alternative to piezo-based sensors for a potential future extension to $\mathrm{KM} 3 \mathrm{NeT}$ are discussed elsewhere [47].

\section{Status and outlook}

Limits on the flux of ultra-high energy neutrinos have been derived by the SPATS [48], SAUND [49] and ACoRNE [50] experiments. While the effective volumes of these experiments are too small to derive competitive limit, it could be shown that the tools and techniques for setting such limits by means of acoustic neutrino detection are in place.

To assess the feasibility of acoustic neutrino detection in a natural body of water, ambient noise and transient background at the installation site have to be investigated. Ambient noise is broadband and mainly caused by agitation of the sea surface [51], i.e. by rain, wind, breaking waves, spray, and cavitation. It is predominantly the ambient background that determines the energy threshold for neutrino detection. Transient noise signals have short duration and an amplitude exceeding the ambient noise level. When of bipolar shape, these signals can mimic pulses from neutrino interactions. Sources of such signals can be anthropogenic, e.g. shipping traffic, or marine fauna. In particular dolphins emit short signals with a spectrum similar to that of acoustic emissions from neutrino interactions.

As measured by several experiments in various natural bodies of water [52-54], the ambient background is low and stable and in general favourable for the operation of an acoustic neutrino detector. The transient background in the Mediterranean Sea has a relatively high level, mainly caused by dolphins and shipping traffic. It is essential to reduce this background completely in order to measure the low expected rate of cosmogenic neutrinos. A strong background reduction is already achieved with methods developed with AMADEUS [55]. For further reduction, in the opinion of this author it is essential to use the specific flat disk-like shape of the signal (cf. Section 2) as a selection criterion [56]. This has not been possible so far due to the small effective volume of existing arrays; such investigations are a main focus for acoustic investigations with the KM3NeT detector.

Lake Baikal has preferable background conditions due to the absence of marine life emitting sound in the relevant frequency range. In addition, the attenuation length is longer in fresh water 
than in salt water [57] and the acoustic sensors of the experiment are located closer to the surface. This allows for an easier separation between signals originating from above and below, with transient background signals more likely coming from the surface. The temperature of Lake Baikal is however only $1.5-2^{\circ} \mathrm{C}$ higher than that for the maximum density of water at a given depth [58], providing for a small value of $\alpha$ in Eq. (2), strongly suppressing the pressure amplitude for a given neutrino energy.

Acoustic activities in Lake Baikal continue while the activities in the Mediterranean Sea concentrate on KM3NeT. With the acoustic background in the Mediterranean Sea being well understood, it seems that efforts should be directed towards reducing the energy threshold for triggering acoustic neutrino events and improving the signal classification. Monte Carlo simulations should be advanced, using up-to-date simulations of the shower development at ultra-high energies for both hadronic and electromagnetic showers. Given the manifold activities on radio detection in ice, as evidenced by the numerous contributions on the subject to the ARENA 2018 workshop, it is the opinion of this author that radio/acoustic hybrid detection has the potential to improve neutrino identification in ice.

\section{Acknowledgements}

The author wishes to thank the organisers of the ARENA 2018 workshop for the invitation to give a presentation. He is also indebted to R. Nahnhauer for making the transparencies from his ARENA 2010 presentation available.

\section{References}

[1] G.A. Askariyan, Sov. J. At. En. 3, 921 (1957), Russian original: At. Energ. 3, 152 (1957)

[2] C.L. Cowan et al., Science 124, 103 (1956)

[3] B.L. Beron and R. Hofstadter, Phys. Rev. Lett. 23, 184 (1969)

[4] G.A. Askariyan et al., Nucl. Inst. and Meth. 164, 267 (1979)

[5] J.G. Learned, Phys. Rev. D 19, 3293 (1979)

[6] L. Sulak et al., Nucl. Inst. and Meth. 161, 203 (1979)

[7] S.D. Hunter et al., J. Acoust. Soc. Am. 69, 1557 (1981)

[8] S.D. Hunter, W.V. Jones, and D.J. Malbrough, J. Acoust. Soc. Am. 69, 1563 (1981)

[9] V.I. Albul et al., Instr. Exp. Tech. 44, 327 (2001)

[10] V.I. Albul et al., Instr. Exp. Tech. 47, 507 (2004)

[11] R. Lahmann et al., Astropart. Phys. 65, 69 (2015)

[12] A. Roberts, Rev. Mod. Phys. 64, 259 (1992)

[13] R. Nahnhauer, in Proc. of ARENA 2010 workshop (2012), Nucl. Inst. and Meth. A 662, p. S20

[14] G.A. Askaryan and B. Dolgoshein, JETP Lett. 25, 213 (1977)

[15] T. Bowen, in Proc. of ICRC 1977 (1977), Vol. 6, p. 277

[16] L.R. Sulak et al., in Proc. of ICRC 1977 (1977), Vol. 11, p. 420

[17] J.G. Learned and R.J.Wilkes for the DUMAND Coll., in Proc. of ICRC 1993 (World Scientific, 1993), Vol. 4, p. 538

[18] A. Capone et al., in Proc. of ICRC 2001 (2001), p. 1264

[19] L.G. Dedenko et al., in Proc. of ICRC 1997 (1997), Vol. 7, p. 89

[20] DUMAND at the Univ. of Hawaii (accessed July 2018), https://www.phys.hawaii.edu/ dumand

[21] S. Bevan et al. (ACoRNE Coll.), Nucl. Inst. and Meth. A 607, 398 (2009)

[22] L.D. Landau and E.M. Lifschitz, Course of Theoretical Physics, Vol.6: Fluid Mechanics, 2nd edn. (Pergamon Press, 1987) 
[23] A.V. Butkevich et al., Phys. Part. Nucl. 29, 266 (1998)

[24] S. Bevan et al. (ACoRNE Coll.), Astropart. Phys. 28, 366 (2007)

[25] P.B. Price, J. of Geophys. Res 111, B02201 (2006), arXiv:astro-ph/0506648v1

[26] G. Manthei, J. Eisenblätter, and T. Spies, in Proc. of ARENA 2005 workshop (World Scientific, 2006), Int. J. Mod. Phys. A21S1, p. 30, ISBN 981-256-755-0

[27] R. Nahnhauer, A.A. Rostovtsev, and D. Tosi, Nucl. Inst. and Meth. A 587, 29 (2008)

[28] S.W. Barwick, Past and Present Development of Radio Cherenkov Neutrino Detectors, in Proc. of ARENA 2018 (2018), to be published in: EPJ Web of Conf.

[29] K. Greisen, Phys. Rev. Lett. 16, 748 (1966)

[30] G.T. Zatsepin and V.A. Kuz'min, 4, 78 (1966), russian original: Pis'ma Zh. Éksp. Teor. Fiz. 4 (1966) 114

[31] V.S. Berezinsky and G.T. Zatsepin, PL B 28, 423 (1969)

[32] A. Achterberg et al. (IceCube Coll.), Astropart. Phys. 26, 155 (2006), arXiv:astro-ph/0604450

[33] M. Ageron et al. (ANTARES Coll.), Nucl. Inst. and Meth. A 656, 11 (2011)

[34] A. Avrorin et al., in Proc. of VLVnT 2009 (2011), Nucl. Inst. and Meth. A 626-627, p. S13

[35] R. Engel, D. Seckel, and T. Stanev, Phys. Rev. D 64, 093010 (2001)

[36] G. Anton et al., Astropart. Phys. 26, 301 (2006)

[37] T. Karg, for the IceCube Coll., Nucl. Inst. and Meth. A 662, S36 (2012)

[38] Y. Abdou et al. (IceCube Coll.), Nucl. Inst. and Meth. A 683, 78 (2012)

[39] N. Budnev, in Proc. of ARENA 2016 (2017), Number 135 in EPJ Web of Conf., p. 06004

[40] S. Aiello et al.(NEMO Coll.), L. Cosentino et al. (2008), arXiv:0804.2913 [astro-ph]

[41] J.A. Aguilar et al. (ANTARES Coll.), Nucl. Inst. and Meth. A 626-627, 128 (2011)

[42] S. Danaher for the ACoRNE Coll. , J. Phys. Conf. Ser. 81, 012011 (2007)

[43] N.G. Lehtinen et al., Astropart. Phys. 17, 279 (2002), arXiv:astro-ph/0104033v1

[44] S. Viola et al., Underwater acoustic positioning system for the SMO and KM3NeT-Italia projects, in Proc. of VLVnT 2013 (2014), AIP Conf. Proc. 1630, p. 134

[45] S. Viola et al., KM3NeT acoustic positioning and detection system, in Proc. of ARENA 2018 (2018), to be published in: EPJ Web of Conf.

[46] S. Adrián-Marínez et al.(KM3NeT Coll.), J. Phys. G: Nucl. Part. Phys. 43, 084001 (2016)

[47] E.J. Buis et al., Fiber optic hydrophones in an acoustic neutrino telescope, in Proc. of ARENA 2018 (2018), to be published in: EPJ Web of Conf.

[48] R. Abbasi et al. (IceCube Coll.), Astropart. Phys. 35, 312 (2012)

[49] N. Kurahashi, J. Vandenbroucke, and G. Gratta, Phys. Rev. D 82, 073006 (2010)

[50] S. Bevan, Nucl. Inst. and Meth. A 604, 143 (2009)

[51] R.J. Urick, Ambient Noise in the Sea (Peninsula publishing, 1986), ISBN 0-932146-13-9

[52] N. Kurahashi and G. Gratta, Phys. Rev. D 78, 092001 (2008)

[53] G. Riccobene for the NEMO Coll., in Proc. ARENA 2008 (2009), Nucl. Inst. and Meth. A 604, p. 149

[54] D. Kießling, Master's thesis, Univ. Erlangen-Nürnberg (2013), ECAP-2013-041

[55] M. Neff, Ph.D. thesis, Univ. Erlangen-Nürnberg (2013), ECAP-2013-023

[56] D. Kießling, Ph.D. thesis, Univ. Erlangen-Nürnberg (2018), to be published

[57] R. Lahmann, in Proc. of ARENA 2016 (2017), Number 135 in EPJ Web of Conf., p. 06001

[58] V. Aynutdinov et al., Nucl. Inst. and Meth. A 662, S210 (2012), doi:10.1016/j.nima.2010.11.153 